\title{
Dental attendance, oral health and the quality of life
}

\author{
Can dental attendance improve quality of life? by C. Mc Grath, and R. Bedi BrDent J 2001; 190: 262-265
}

\begin{abstract}
Objective
The aim of this study was to determine the relationship between reported dental attendance patterns and the public's perception of how oral health impacts on quality of life (QoL).
\end{abstract}

\begin{abstract}
Method
A national UK study involving a random probability sample of 2,668 adults. Respondents were interviewed in their homes about how oral health affects their QoL and about their dental attendance pattern. Responses were coded as oral health having a negative impact, positive impact or impact in general (either positive and/or negative) on QoL.

\section{Results}

The response rate was $70 \%$ with 1,865 adults participating in the study. $72 \%(1,340)$ reported that their oral health affected their QoL in general, $57 \%(1,065)$ reported that it had a positive effect, and $48 \%(902)$ that it had a negative effect. $61 \%(1,136)$ reported to have attended the dentist within the last year - 'regular attenders'. Bivaraite analysis identified association between perception of how oral health impacts on QoL and dental attendance pattern $(P<0.01)$. When socio-demographic factors (age, gender, and social class) were taken into account in the
\end{abstract}

analysis, 'regular attenders' reported that oral health had greater impact in general on $\mathrm{QoL}(\mathrm{OR}=1.30,95 \% \mathrm{CI}=1.04,1.63)$ and, specifically, a greater positive impact $(\mathrm{OR}=1.49,95 \%$ $\mathrm{CI}=1.44,1.77)$.

\section{Conclusion}

Dental attendance is associated with perceptions of how oral health impacts on QoL, specifically enhanced life quality. This may have implications for understanding the health gain of regular dental attendance.

\section{In brief}

- Seventy-two per cent of respondents from a national survey reported that oral health affected their quality of life

- The majority reported that this was a positive effect

- Regular dental attenders reported that oral health had a greater impact upon life quality

- Dental attendance is associated with perceptions on how oral health impacts upon quality of life, specifically enhanced life quality

\begin{abstract}
Comment
Traditional clinical indicators such as 1 DMFT and CPITN measure oral disease rather than oral health which is a multi-dimensional concept incorporating social and psychological dimensions. Considerable interest in measurement of the impact of oral health on daily living has led to the recent development of at least 11 different indicators of oral health related quality of life. Most were developed for use in older people and few have been evaluated among UK populations.

This paper set out to determine the relationship between dental attendance patterns and perceptions of the impact of oral health on quality of life. The study used face to face interviews carried out as part of the Office for National Statistics (ONS) Omnibus Survey in Great Britain, benefiting from their sampling expertise and skilled professional interviewers. Unlike most previous studies an entirely qualitative approach was used for this research and
\end{abstract}

both positive and negative influences of oral health were captured. Regular dental attenders, defined as people who had visited a dentist within the past year, were significantly more likely to perceive the impacts of oral health on quality of life, particularly the positive effects. This association remained after controlling for confounding factors such as age, gender and social class.

One interesting finding from this study was the lack of any association between irregular attendance and negative oral health impacts. This might have resulted from the definition of regular attendance used and contrasts with the findings of a number of other studies involving measures of negative impact such as the Oral Health Impact Profile (OHIP). ${ }^{1}$ The 1998 UK Adult Dental Health Survey, ${ }^{2}$ also conducted by ONS, showed that symptomatic attenders were significantly more likely to report impacts included in a shortened version of the OHIP than those seeking check-ups on a regular or occasional basis.

This paper provides some useful evidence to support the benefits of regular dental care but, as the authors rightly point out, their findings have only demonstrated an association between dental attendance and quality of life. Further research involving a longitudinal design will be required in order to answer the question posed in the title of the paper.

\section{Mike Prendergast}

Consultant in Dental Public Health, Shropshire Health Authority

1 Slade G D, Spencer A J. Development and evaluation of the oral health impact profile. Community Dent Health 1994; 11: 3-11.

2 Kelly M, Steele J et al. Adult Dental Health Survey - Oral Health in the United Kingdom 1998. London: The Stationery Office, 2000. 